\title{
Session IV
}

\section{Nucleosynthesis and Yields}




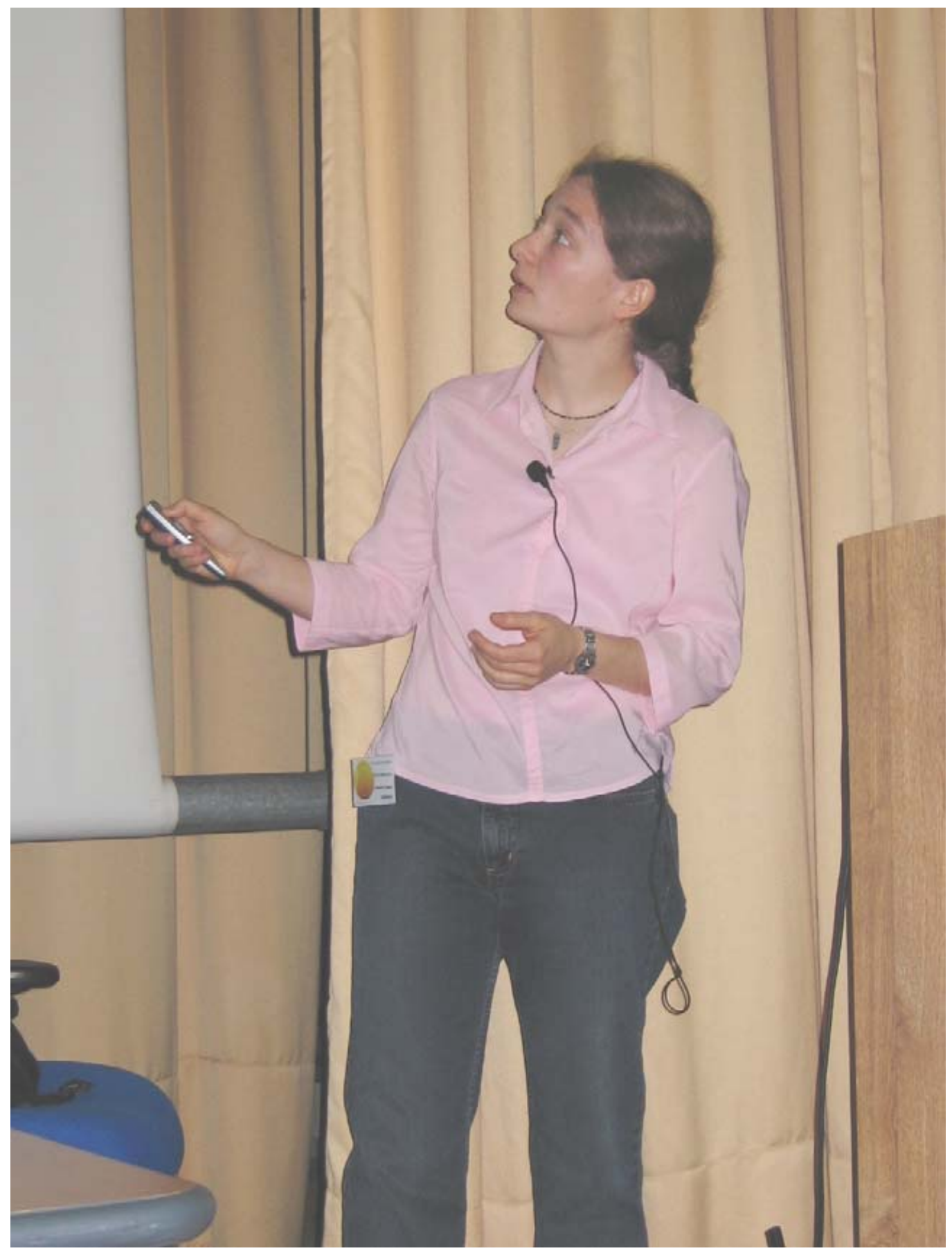

Carla Frölich exposing a possible supernovae explosion mechanism. 


\title{
Yields of Population III Supernovae and the Abundance Patterns of Extremely Metal-Poor Stars
}

\author{
K. Nomoto ${ }^{1}$, N. Tominaga ${ }^{1}$, H. Umeda ${ }^{1}$, C. Kobayashi ${ }^{2}$ \\ ${ }^{1}$ Department of Astronomy, University of Tokyo, Bunkyo-ku, Tokyo 113-0033, Japan \\ email: nomoto@astron.s.u-tokyo.ac.jp \\ ${ }^{2}$ Max-Planck-Institut für Astrophysik, Garching, Germany
}

\begin{abstract}
The abundance patterns of extremely metal-poor (EMP) stars provide us with important information on nucleosynthesis in supernovae (SNe) formed in a Pop III or EMP environment, and thus on the nature of the first stars in the Universe. We review nucleosynthesis yields of various types of those SNe, focusing on core-collapse (black-hole-forming) SNe with various progenitor masses, explosion energies (including Hypernovae), and asphericity. We discuss the implications of the observed trends in the abundance ratios among iron-peak elements, and the large $\mathrm{C} / \mathrm{Fe}$ ratio observed in certain EMP stars with particular attention to recently discovered hyper metal-poor (HMP) stars. We show that the abundance pattern of the HMP stars with $[\mathrm{Fe} / \mathrm{H}]<-5$ and other EMP stars are in good accord with those of black-hole-forming supernovae, but not pair-instability supernovae. This suggests that black-hole-forming supernovae made important contributions to the early Galactic (and cosmic) chemical evolution. Finally we discuss the nature of First (Pop III) Stars.
\end{abstract}

Keywords. Nuclear reactions, nucleosynthesis, abundances, stars: Population II, supernovae

\section{The First Stars and Metal-Poor Stars}

It is of vital importance to identify the first generation stars in the Universe, i.e., totally metal-free $(Z=0)$, Population (Pop) III stars. The impact of the formation of Pop III stars on the evolution of the Universe depends on their typical masses. Recent numerical models have shown that, the first stars are as massive as $\sim 100 M_{\odot}$ Abel et al. 2002. The formation of long-lived low mass Pop III stars may be inefficient because of slow cooling of metal-free gas cloud, which is consistent with the failure of attempts to find Pop III stars. However, it is still controversial how the initial mass function (IMF) depends on the metallicity.

In the early universe, the chemical enrichment by a single SN can dominate the preexisting metal contents, because the timescale of mixing in the interstellar medium may be longer than the age of the Galaxy. Thus the abundance pattern of the enriched gas may reflect nucleosynthesis in the single SN (Audouze \& Silk 1995, Ryan et al. 1996, Shigeyama \& Tsujimoto 1998, Nakamura et al. 1999). Low-mass stars formed in the chemically enriched gas have long lifetimes and are observed as Extremely Metal-Poor (EMP) stars and Hyper Metal-Poor (HMP) stars (Beers \& Christlieb 2005). Therefore the abundance patterns of EMP and HMP stars reflect the yield of a single SN at extremely metal-poor environment, thus constraining the nature of the first (Pop III) SNe and IMF.

Here we summarize the comparisons between the abundance patterns of the EMP stars (Cayrel et al. 2004, Honda et al. 2004) and HMP stars, HE0107-5240 (Christlieb et al. 2002) and HE1327-2326 (Frebel et al. 2005). 

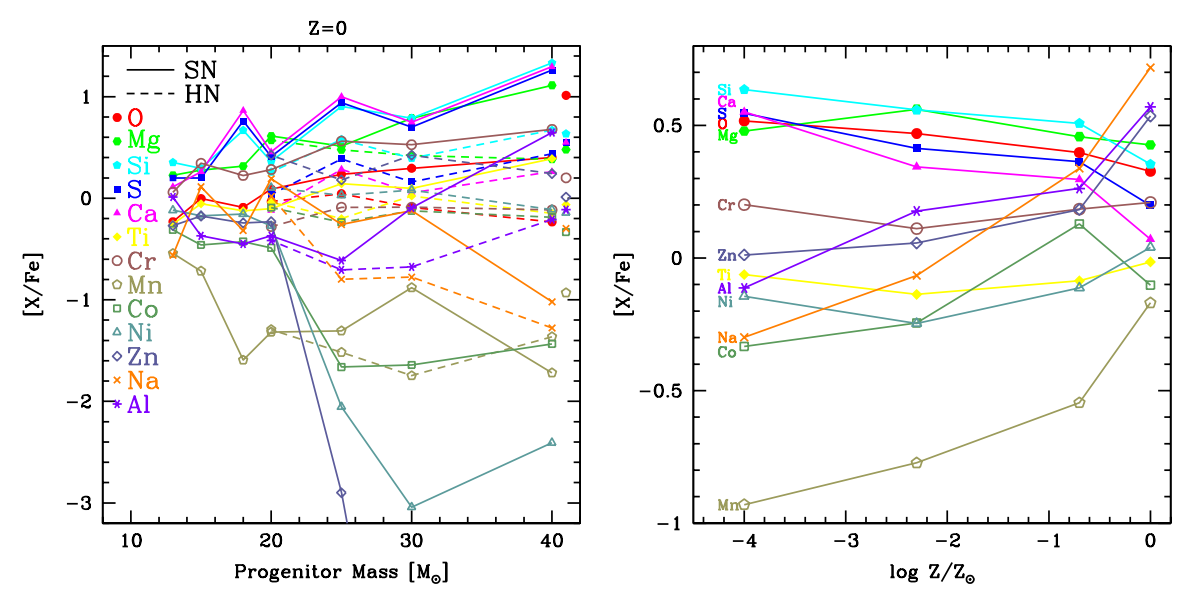

Figure 1. (left) Relative abundance ratios as a function of progenitor mass with $Z=0$. The solid and dashed lines show normal SNe II with $E_{51}=1$ and HNe. (right) The IMF weighted abundance ratios as a function of metallicity of progenitors, where the $\mathrm{HN}$ fraction $\epsilon_{\mathrm{HN}}=0.5$ is adopted. $Z=0$ results are plotted at $\log Z / Z_{\odot}=-4$.

\section{Nucleosynthesis and Metallicity}

We have calculated the chemical yields for various metallicities, including Hypernovae (HNe: see next section) (Tominaga et al. 2005).

In the metal-free stellar evolution, because of the lack of initial CNO elements, the CNO cycle does not operate until the star contracts to a much higher central temperature $\left(\sim 10^{8} \mathrm{~K}\right)$ than population II stars, where the $3 \alpha$ reaction produces a tiny fraction of ${ }^{12} \mathrm{C}$ $\left(\sim 10^{-10}\right.$ in mass fraction). However, the late core evolution and the resulting Fe core masses of metal-free stars are not significantly different from metal-rich stars. Therefore, $[\alpha / \mathrm{Fe}]$ is larger by only a fraction of $\sim 0.2$ dex and the abundance ratios of the iron-peak elements are not so different from metal-rich stars, except for Mn. On the other hand, the CNO cycle produces only a small amount of ${ }^{14} \mathrm{~N}$, which is transformed into ${ }^{22} \mathrm{Ne}$ during He-burning. The surplus of neutrons in ${ }^{22} \mathrm{Ne}$ increases the abundances of odd-Z elements $(\mathrm{Na}, \mathrm{Al}, \mathrm{P}, \ldots)$. Therefore, the metallicity effect is realized for odd- $\mathrm{Z}$ elements and the inverse ratio of $\alpha$ elements and their isotopes (e.g., $\left.{ }^{13} \mathrm{C} /{ }^{12} \mathrm{C}\right) .[\mathrm{Na} / \mathrm{Fe}]$ and $[\mathrm{Al} / \mathrm{Fe}]$ of metal-free stars are smaller by $\sim 1.0$ and 0.7 dex than solar abundance stars, which are consistent with the observed trends.

Figure 1 shows the abundance ratios of Pop III $(Z=0)$ SNe II and HNe as a function of the progenitor mass (left) and the IMF weighted yields of SNe II and HNe as a function of metallicity (right).

The solid and dashed lines show the SN II and HN yields, respectively. The yield masses of $\alpha$ elements (O, Ne, Mg, Si, S, Ar, Ca, and Ti) are larger for more massive stars because of the larger mantle mass. Since the Fe mass is $\sim 0.1 M_{\odot}$, being independent of the progenitor's mass for $E_{51}=1$, the abundance ratio $[\alpha / \mathrm{Fe}]$ is larger for more massive stars. For HNe, although Fe production is larger for more massive stars because of the higher energy, $[\alpha / \mathrm{Fe}]$ is almost constant independent of the stellar mass because we assume the mass-cut to get $[\mathrm{O} / \mathrm{Fe}]=0.4$. 

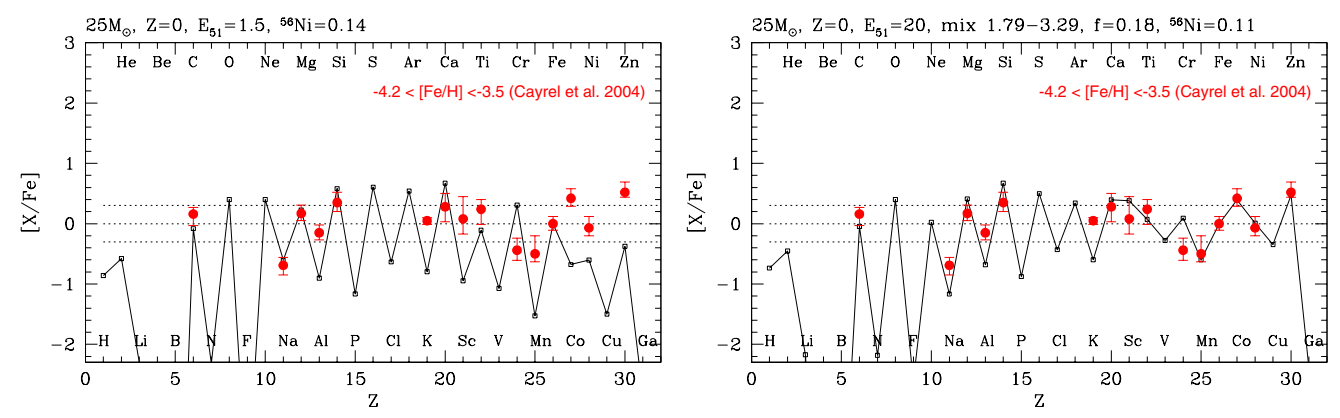

Figure 2. Averaged elemental abundances of stars with $[\mathrm{Fe} / \mathrm{H}]=-3.7$ (Cayrel et al. 2004) compared with the normal SN yield (left: $25 M_{\odot}, E_{51}=1.5$ ), and the hypernova yield (right: $\left.25 M_{\odot}, E_{51}=20\right)$.

\section{Hypernova Yields}

Regarding supernova (SN) yields, one of the most interesting recent developments is the discovery of hyper-energetic supernovae, whose kinetic energy (KE) exceeds $10^{52} \mathrm{erg}$, about 10 times the $\mathrm{KE}$ of normal core-collapse $\mathrm{SNe}$ (hereafter $E_{51}=E / 10^{51} \mathrm{erg}$ ) (Nomoto et al. 2004). Such SNe are called Hypernovae (HNe). Nucleosynthesis in HNe has some special features, so that the contribution of HNe in early chemical evolution can be seen the abundance patterns of HMP/EMP stars.

\subsection{Hypernovae and Faint Supernovae}

The Hypernovae 1998bw and 2003dh were clearly linked to the Gamma-Ray Bursts GRB 980425 (Galama et al. 1998) and GRB 030329 (Stanek et al. 2003; Hjorth et al. 2003), thus establishing the connection between long GRBs and core-collapse supernovae (SNe). SNe 1998bw and 2003dh were exceptional for SNe Ic: they were as luminous at peak as a SN Ia, indicating that they synthesized $0.3-0.5 M_{\odot}$ of ${ }^{56} \mathrm{Ni}$, and their kinetic energy (KE) were estimated as $E_{51} \sim 30-50$ (Iwamoto et al. 1998; Woosley et al. 1999; Nakamura et al. 2001a).

Other HNe have been recognized, such as SN 1997ef (Iwamoto et al. 2000; Mazzali, Iwamoto, \& Nomoto 2000) and SN 2002ap (Mazzali et al. 2002). These hypernovae span a wide range of properties, although they all appear to be highly energetic compared to normal core-collapse SNe. The mass estimates, obtained from fitting the optical light curves and spectra, place hypernovae at the high-mass end of SN progenitors.

In contrast, SNe II 1997D and 1999br were very faint SNe with very low KE (Turatto et al. 1998; Hamuy 2003; Zampieri et al. 2003). In the diagram that shows $E$ and the mass of ${ }^{56} \mathrm{Ni}$ ejected $M\left({ }^{56} \mathrm{Ni}\right)$ as a function of the main-sequence mass $M_{\mathrm{ms}}$ of the progenitor star, therefore, we propose that SNe from stars with $M_{\mathrm{ms}} \gtrsim 20-25 M_{\odot}$ have different $E$ and $M\left({ }^{56} \mathrm{Ni}\right)$, with a bright, energetic "hypernova branch" at one extreme and a faint, low-energy SN branch at the other (Nomoto et al. 2003). For the faint SNe, the explosion energy was so small that most ${ }^{56} \mathrm{Ni}$ fell back onto the compact remnant. The extreme cases of the faint SN branch may correspond to HMP stars as will be discussed below. Between the two branches, there may be a variety of SNe (Hamuy 2003).

This trend might be interpreted as follows. Stars more massive than $\sim 25 M_{\odot}$ form a black hole at the end of their evolution. Stars with non-rotating black holes are likely to collapse "quietly" ejecting a small amount of heavy elements (faint supernovae). In contrast, stars with rotating black holes are likely to give rise to Hypernovae. The hypernova progenitors might form the rapidly rotating cores by spiraling-in of a companion star in a binary system. 


\subsection{Nucleosynthesis in Hypernovae}

In core-collapse supernovae/hypernovae, stellar material undergoes shock heating and subsequent explosive nucleosynthesis. Iron-peak elements are produced in two distinct regions, which are characterized by the peak temperature, $T_{\text {peak }}$, of the shocked material. For $T_{\text {peak }}>5 \times 10^{9} \mathrm{~K}$, material undergoes complete Si burning whose products include $\mathrm{Co}, \mathrm{Zn}, \mathrm{V}$, and some $\mathrm{Cr}$ after radioactive decays. For $4 \times 10^{9} \mathrm{~K}<T_{\text {peak }}<5 \times 10^{9} \mathrm{~K}$, incomplete Si burning takes place and its after decay products include $\mathrm{Cr}$ and $\mathrm{Mn}$ (e.g., Nakamura et al. 1999).

We note the following characteristics of nucleosynthesis with very large explosion energies (Nakamura et al. 2001b; Nomoto et al. 2001; Umeda \& Nomoto 2005):

(1) Both complete and incomplete Si-burning regions shift outward in mass compared with normal supernovae, so that the mass ratio between the complete and incomplete Si-burning regions becomes larger. As a result, higher energy explosions tend to produce larger $[(\mathrm{Zn}, \mathrm{Co}, \mathrm{V}) / \mathrm{Fe}]$ and smaller $[(\mathrm{Mn}, \mathrm{Cr}) / \mathrm{Fe}]$, which can explain the trend observed in very metal-poor stars (Umeda \& Nomoto 2005).

(2) In the complete Si-burning region of hypernovae, elements produced by $\alpha$-rich freezeout are enhanced. Hence, elements synthesized through capturing of $\alpha$-particles, such as ${ }^{44} \mathrm{Ti},{ }^{48} \mathrm{Cr}$, and ${ }^{64} \mathrm{Ge}$ (decaying into ${ }^{44} \mathrm{Ca},{ }^{48} \mathrm{Ti}$, and ${ }^{64} \mathrm{Zn}$, respectively) are more abundant.

Hypernova nucleosynthesis may have made an important contribution to Galactic chemical evolution. In the early galactic epoch when the galaxy was not yet chemically well-mixed, $[\mathrm{Fe} / \mathrm{H}]$ may well be determined by mostly a single SN event (Audouze \& Silk 1995). The formation of metal-poor stars is supposed to be driven by a supernova shock, so that $[\mathrm{Fe} / \mathrm{H}]$ is determined by the ejected Fe mass and the amount of circumstellar hydrogen swept-up by the shock wave (Ryan, Norris, \& Beers 1996). Then, hypernovae with larger $E$ are likely to induce the formation of stars with smaller $[\mathrm{Fe} / \mathrm{H}]$, because the mass of interstellar hydrogen swept up by a hypernova is roughly proportional to $E$ (Ryan et al. 1996; Shigeyama \& Tsujimoto 1998) and the ratio of the ejected iron mass to $E$ is smaller for hypernovae than for normal supernovae.

\section{Extremely Metal-Poor (EMP) Stars}

Here we make comparisons between the theoretical yields and the abundance patterns of the EMP stars, given in Cayrel et al. (2004) and Honda et al. (2004) where the data of 35 and 22 metal-poor stars are provided, respectively. Though the trends of elemental abundance ratios are similar to those found earlier (McWilliam et al. 1995, Ryan et al. 1996), Cayrel et al. (2004) found much smaller dispersions.

\subsection{Averaged Abundance Patterns of EMP Stars}

First the theoretical yields are compared with the averaged abundance pattern of four EMP stars, CS 22189-009, CD-38:245, CS 22172-002 and CS 22885-096, which have low metallicities $(-4.2<[\mathrm{Fe} / \mathrm{H}]<-3.5)$ and normal $[\mathrm{C} / \mathrm{Fe}] \sim 0$ (Cayrel et al. 2004). Figures 2 show the comparison with the $25 M_{\odot}$ model of normal energy explosion $\left(E_{51}=\right.$ 1.5: left) and hyper energetic explosion $\left(E_{51}=20\right.$ : right $)$.

In the normal SN model, the mass-cut is determined to eject Fe of mass $\left.0.14 M_{\odot}\right)$. Then the yields are in reasonable agreements with the observations for the ratios [(Na, $\mathrm{Mg}, \mathrm{Si}) / \mathrm{Fe}]$, but give too small $[(\mathrm{Sc}, \mathrm{Ti}, \mathrm{Mn}, \mathrm{Co}, \mathrm{Ni}, \mathrm{Zn}) / \mathrm{Fe}]$ and too large $[(\mathrm{Ca}, \mathrm{Cr}) / \mathrm{Fe}]$.

In the HN model (right), these ratios are in much better agreement with observations. The ratios of $\mathrm{Co} / \mathrm{Fe}$ and $\mathrm{Zn} / \mathrm{Fe}$ are larger in higher energy explosions since both $\mathrm{Co}$ and $\mathrm{Zn}$ are synthesized in complete Si burning at high temperature region. To account for 

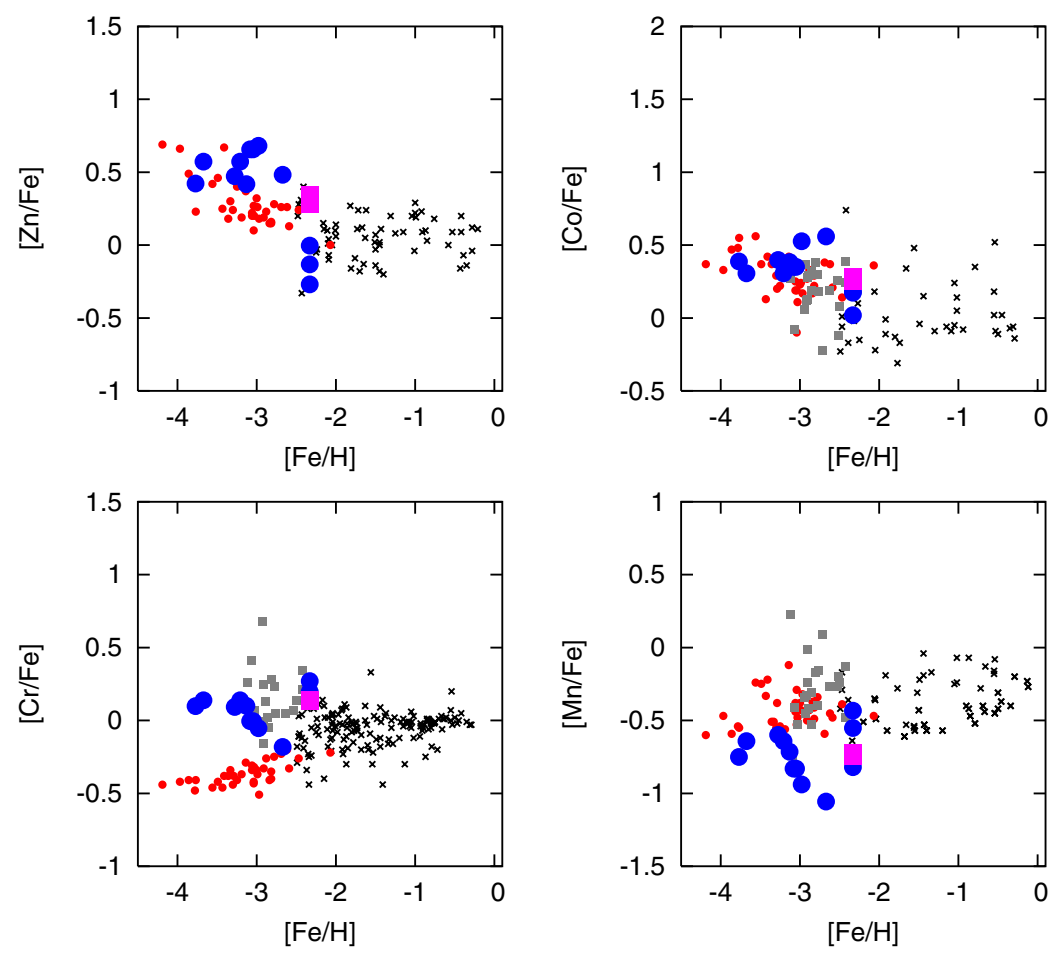

Figure 3. The comparison between the $[\mathrm{Zn}, \mathrm{Co}, \mathrm{Cr}, \mathrm{Mn} / \mathrm{Fe}]$ trends of observed stars (the previous studies cross, Cayrel et al. (2004) red circle, Honda et al. (2004) gray square), and those of individual stars models (blue circle) and IMF integration (large pink square).

the observations, materials synthesized in a deeper complete Si-burning region should be ejected, but the amount of Fe should be small. This is realized in the mixing-fallback models (Umeda \& Nomoto 2002, 2005).

In the HN model (right), the low-density progenitor model is used (Umeda \& Nomoto $2005)$ to enhance the $\alpha$-rich freeze-out. As a result, the $\mathrm{Sc} / \mathrm{Fe}$ and $\mathrm{Ti} / \mathrm{Fe}$ ratios are especially enhanced.

\subsection{Hypernovae and the Abundance Trends}

It has been found that, in the observed abundances of halo stars, there are significant differences between the abundance patterns in the iron-peak elements below and above $[\mathrm{Fe} / \mathrm{H}] \sim-2.5--3$.

(1) For $[\mathrm{Fe} / \mathrm{H}] \lesssim-2.5$, the mean values of $[\mathrm{Cr} / \mathrm{Fe}]$ and $[\mathrm{Mn} / \mathrm{Fe}]$ decrease toward smaller metallicity, while $[\mathrm{Co} / \mathrm{Fe}]$ increases (McWilliam et al. 1995; Ryan et al. 1996). Cayrel et al. (2004) found much flatter trend of $[\mathrm{Mn} / \mathrm{Fe}]$.

(2) $[\mathrm{Zn} / \mathrm{Fe}] \sim 0$ for $[\mathrm{Fe} / \mathrm{H}] \simeq-3$ to 0 (Sneden, Gratton, \& Crocker 1991), while at $[\mathrm{Fe} / \mathrm{H}]<-3.3,[\mathrm{Zn} / \mathrm{Fe}]$ increases toward smaller metallicity (Cayrel et al. 2004).

The larger $[(\mathrm{Zn}, \mathrm{Co}) / \mathrm{Fe}]$ and smaller $[(\mathrm{Mn}, \mathrm{Cr}) / \mathrm{Fe}]$ in the supernova ejecta can be realized if the mass ratio between the complete Si burning region and the incomplete Si burning region is larger, or equivalently if deep material from the complete Si-burning region is ejected by mixing or aspherical effects. This can be realized if (1) the mass cut between the ejecta and the compact remnant is located at smaller $M_{r}$ (Nakamura et al. 1999), (2) $E$ is larger to move the outer edge of the complete Si burning region to larger $M_{r}$ (Nakamura et al. 2001b), or (3) asphericity in the explosion is larger. 

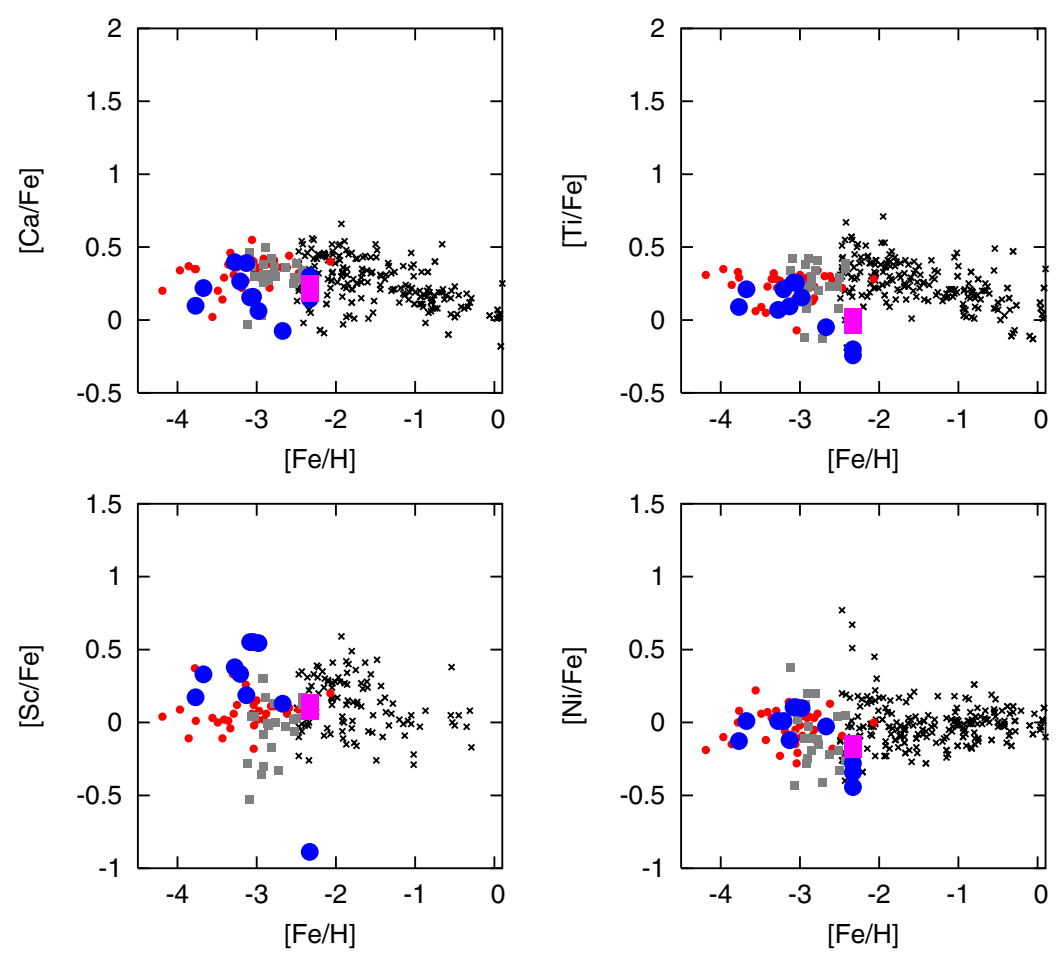

Figure 4. The comparison between the $[\mathrm{Ca}, \mathrm{Ti}, \mathrm{Sc}, \mathrm{Ni} / \mathrm{Fe}]$ trends of observed stars (the previous studies cross, Cayrel et al. (2004) red circle, Honda et al. (2004) gray square) and those of individual stars models (blue circle) and IMF integration (large pink square).

Among these possibilities, a large explosion energy $E$ enhances $\alpha$-rich freezeout, which results in an increase of the local mass fractions of $\mathrm{Zn}$ and $\mathrm{Co}$, while $\mathrm{Cr}$ and $\mathrm{Mn}$ are not enhanced (Umeda \& Nomoto 2002). Models with $E_{51}=1$ do not produce sufficiently large $[\mathrm{Zn} / \mathrm{Fe}]$. To be compatible with the observations of $[\mathrm{Zn} / \mathrm{Fe}] \sim 0.5$, the explosion energy must be much larger, i.e., $E_{51} \gtrsim 20$ for $M \gtrsim 20 M_{\odot}$, i.e., hypernova-like explosions of massive stars $\left(M \gtrsim 25 M_{\odot}\right)$ with $E_{51}>10$ are responsible for the production of $\mathrm{Zn}$.

In the hypernova models, moreover, the overproduction of $\mathrm{Ni}$, as found in the simple "deep" mass-cut model (1), can be avoided (Umeda \& Nomoto 2005). Therefore, if hypernovae made significant contributions to the early Galactic chemical evolution, it could explain the large $\mathrm{Zn}$ and Co abundances and the small $\mathrm{Mn}$ and $\mathrm{Cr}$ abundances observed in very metal-poor stars.

Figures 3-5 show the theoretical trial to reproduce the observed trend in Cayrel et al. (2004) and Honda et al. (2004) with the supernova-induced star formation model. The supernova models with $\left(M / M_{\odot}, E_{51}\right)=(13,1.5),(15,1.5),(18,1.5),(20,10),(25,20)$, $(30,30),(40,40)$, and $(50,50)$ are used, where the stars more massive than $20 M_{\odot}$ are assumed to explode as HNe. These models are located at $[\mathrm{Fe} / \mathrm{H}]=\log _{10}\left(\mathrm{Fe} / E_{51}\right)-C$, and can be consistent with the observed trend even without mixing of materials in the interstellar medium. Since HNe may explode as jet-like explosions, the HN models adopt the mixing-fallback and the "low-density" model (see Umeda \& Nomoto 2005; Tominaga et al. 2005 for details).

The trends of most elements can be well reproduced by these models, except for the overproduction of $\mathrm{Cr}$ and the underproduction of $\mathrm{K}$. For Cr, only the gradient of $\mathrm{Cr}$ with $[\mathrm{Fe} / \mathrm{H}]$ can be reproduced. 

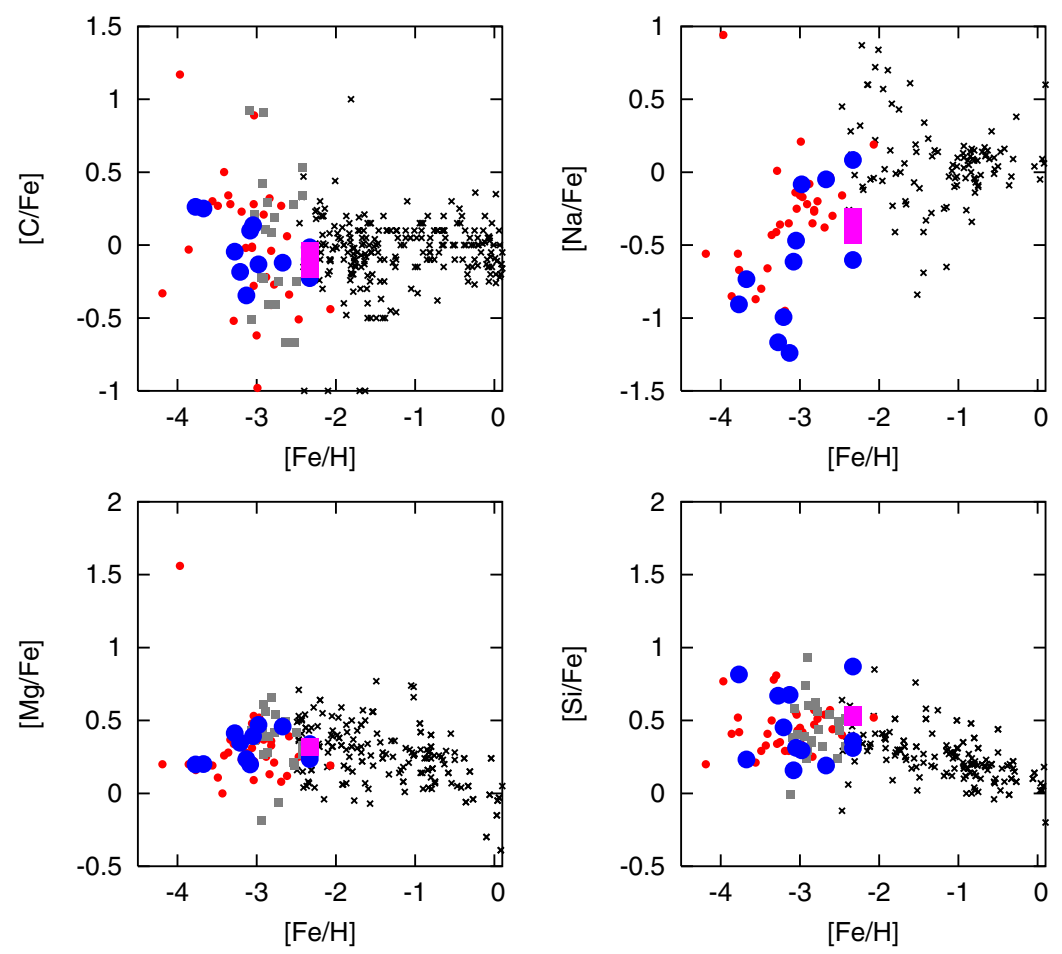

Figure 5. The comparison between the $[\mathrm{C}, \mathrm{Na}, \mathrm{Mg}, \mathrm{Si} / \mathrm{Fe}]$ trends of observed stars (the previous studies cross, Cayrel et al. (2004) red circle, and Honda et al. (2004) gray square) and those of individual stars models (blue circle) and IMF integration (large pink square).

\section{Hyper Metal-Poor (HMP) Stars}

Recently two HMP stars, HE0107-5240 (Christlieb et al. 2002) and HE1327-2326 (Frebel et al. 2005), were discovered, whose metallicity $\mathrm{Fe} / \mathrm{H}$ is smaller than 1/100,000 of the Sun (i.e., $[\mathrm{Fe} / \mathrm{H}]<-5$ ), being more than a factor of 10 smaller than previously known EMP stars. These discoveries have raised an important question as to whether the observed low mass $\left(\sim 0.8 M_{\odot}\right)$ HMP stars are actually Pop III stars, or whether these HMP stars are the second generation stars being formed from gases which were chemically enriched by a single first generation supernova (SN) (Umeda \& Nomoto 2003). This is related to the questions of how the initial mass function depends on the metallicity. Thus identifying the origin of these HMP stars is indispensable to the understanding of the earliest star formation and chemical enrichment history of the Universe.

\subsection{Abundance Patterns of HE0107-5240 8 HE1327-2326}

The elemental abundance patterns of these HMP stars provide a key to the answer to the above questions. The abundance patterns of HE1327-2326 and HE0107-5240 are quite unusual (Fig. 6). The striking similarity of $[\mathrm{Fe} / \mathrm{H}](=-5.4$ and -5.2 for HE13272326 and HE0107-5240, respectively) and $[\mathrm{C} / \mathrm{Fe}](\sim+4)$ suggests that similar chemical enrichment mechanisms operated in forming these HMP stars. However, the N/C and $(\mathrm{Na}, \mathrm{Mg}, \mathrm{Al}) / \mathrm{Fe}$ ratios are more than a factor of 10 larger in HE1327-2326. In order for the theoretical models to be viable, these similarities and differences should be explained self-consistently.

Iwamoto et al. (2005) showed that the above similarities and variations of the HMP stars can be well reproduced in unified manner by nucleosynthesis in the core-collapse 

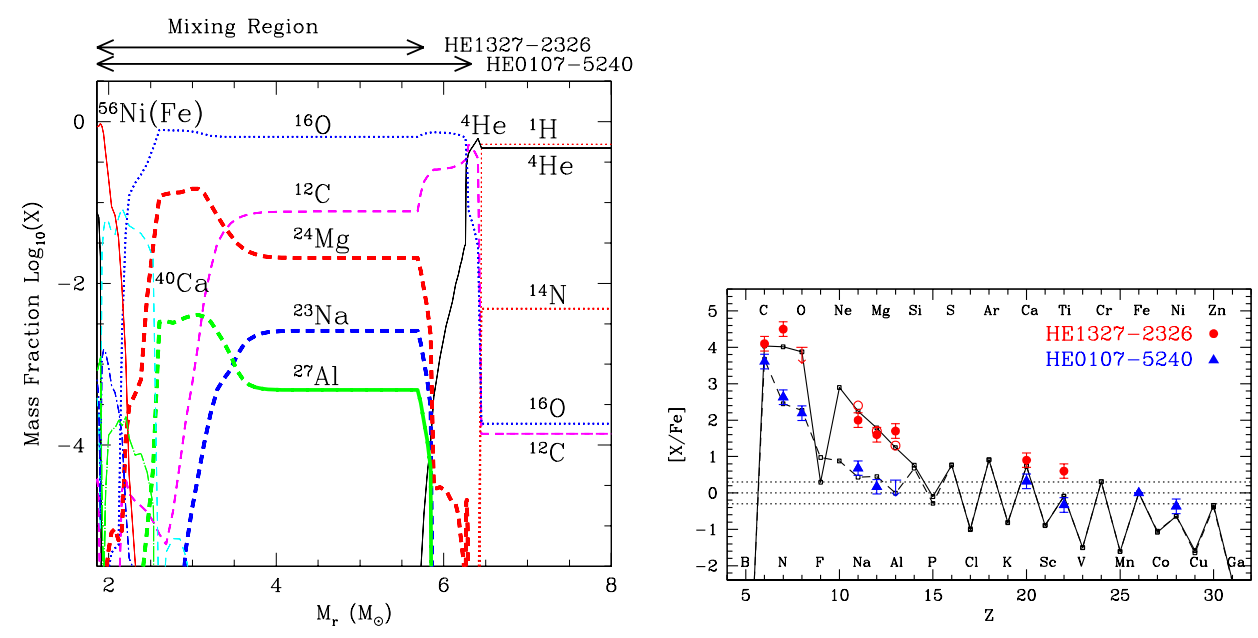

Figure 6. (left) The post-explosion abundance distributions for the $25 M_{\odot}$ model with the explosion energy $E_{51}=0.7$ (Iwamoto et al. 2005). (right) Elemental abundances of HMP stars (filled circles and triangle, compared with a theoretical supernova yield (Iwamoto et al. 2003, 2005).

"faint" supernovae (SNe) which undergo mixing-and-fallback (Umeda \& Nomoto 2003). We thus argue that the HMP stars are the second generation low mass stars, whose formation was induced by the first generation (Pop III) SN with efficient cooling of carbon-enriched gases.

\subsection{Models for HE0107-5240 \& HE1327-2326}

We consider a model that C-rich EMP stars are produced in the ejecta of (almost) metalfree supernova mixed with extremely metal-poor interstellar matter. The similarity of $[\mathrm{Fe} / \mathrm{H}]$ and $[\mathrm{C} / \mathrm{Fe}]$ suggests that the progenitor's masses of Pop III SNe were similar for these HMP stars. We therefore choose the Pop III $25 M_{\odot}$ models and calculate their evolution and explosion (Umeda \& Nomoto 2003, Iwamoto et al. 2005).

The abundance distribution after explosive nucleosynthesis is shown in Figure 6 (left) for the kinetic energy $E$ of the ejecta $E_{51} \equiv E / 10^{51} \mathrm{erg}=0.74$. The abundance distribution for $E_{51}=0.71$ is similar. In the "faint" SN model, most part of materials that underwent explosive nucleosynthesis are decelerated by the influence of the gravitational pull (Woosely \& Weaver 1995; Iwamoto et al. 2005) and will eventually fall back onto the central compact object. The explosion energies of $E_{51}=0.74$ and 0.71 lead to the mass cut $M_{\text {cut }}=5.8 M_{\odot}$ and $6.3 M_{\odot}$, respectively, and the former and the latter models are used to explain the abundance patterns of HE1327-2326 and HE0107-5240, respectively.

During the explosion, the SN ejecta is assumed to undergo mixing, i.e., materials are first uniformly mixed in the mixing-region extending from $M_{r}=1.9 M_{\odot}$ to the mass cut at $M_{r}=M_{\text {cut }}$ (where $M_{r}$ is the mass coordinate and stands for the mass interior to the radius $r$ ) as indicated in Figure 6 (left), and only a tiny fraction, $f$, of the mixed material is ejected from the mixing-region together with all materials at $M_{r}>M_{\text {cut }}$; most materials interior to the mass cut fall back onto the central compact object. Such a mixing-fallback mechanism (which might mimic a jet-like explosion) is required to extract Fe-peak and other heavy elements from the deep fallback region into the ejecta (Umeda \& Nomoto 2003, 2005).

Figure 6 (right) shows the calculated abundance ratios in the SN ejecta models for suitable choice of $f$ which are respectively compared with the observed abundances of 
the two HMP stars. To reproduce $[\mathrm{C} / \mathrm{Fe}] \sim+4$ and other abundance ratios of HMP stars in Figure 6 (right), the ejected mass of Fe is only $1.0 \times 10^{-5} M_{\odot}$ for HE1327-2326 and $1.4 \times 10^{-5} M_{\odot}$ for HE0107-5240. These SNe are much fainter in the radioactive tail than the typical SNe and form massive black holes of $\sim 6 M_{\odot}$.

The question is what causes the large difference in the amount of Na-Mg-Al between the SNe that produced HE0107-5240 and HE1327-2326. Because very little Na-Mg-Al is ejected from the mixed fallback materials (i.e., $f \sim 10^{-4}$ ) compared with the materials exterior to the mass cut, the ejected amount of $\mathrm{Na}-\mathrm{Mg}-\mathrm{Al}$ is very sensitive to the location of the mass cut. As indicated in Figure $6, M_{\text {cut }}$ is smaller (i.e., the fallback mass is smaller) in the model for HE1327-2326 $\left(M_{\text {cut }}=5.8 M_{\odot}\right)$ than HE0107-5240 $\left(M_{\text {cut }}=6.3 M_{\odot}\right)$, so that a larger amount of Na-Mg-Al is ejected from the SN for HE1327-2326. Since $M_{\text {cut }}$ is sensitively determined by the explosion energy, the $(\mathrm{Na}-\mathrm{Mg}-\mathrm{Al}) / \mathrm{Fe}$ ratios among the HMP stars are predicted to show significant variations and can be used to constrain $E_{51}$. Note also that the explosion energies of these SN models with fallback are not necessarily very small (i.e., $E_{51} \sim 0.7$ ). Further these explosion energies are consistent with those observed in the actual "faint" SNe (Turatto et al. 1998).

The next question is why HE1327-2326 has a much larger N/C ratio than HE01075240 . In our models, a significant amount of $\mathrm{N}$ is produced by the mixing between the He convective shell and the H-rich envelope during the presupernova evolution (Umeda et al. 2000 ), where $\mathrm{C}$ created by the triple- $\alpha$ reaction is burnt into $\mathrm{N}$ through the $\mathrm{CN}$ cycle. For the HE1327-2326 model, we assume about 30 times larger diffusion coefficients (i.e., faster mixing) for the $\mathrm{H}$ and $\mathrm{He}$ convective shells to overcome an inhibiting effect of the mean molecular weight gradient (and also entropy gradient) between $\mathrm{H}$ and He layers. Thus, larger amounts of protons are carried into the He convective shell. Then [C/N] $\sim 0$ is realized as observed in HE1327-2326. Such an enhancement of mixing efficiency has been suggested to take place in the present-day massive stars known as fast rotators, which show various $\mathrm{N}$ and He enrichments due to different rotation velocities (Heger \& Woosley 2002).

\section{First Stars}

Recent numerical models have shown that, the first stars are as massive as $\sim 100 M_{\odot}$ (Abel et al. 2002). The formation of long-lived low mass Pop III stars may be inefficient because of slow cooling of metal free gas cloud, which is consistent with the failure of attempts to find Pop III stars.

If the most Fe deficient star, HE0107-5240, is a Pop III low mass star that has gained its metal from a companion star or interstellar matter (Yoshii 1981), would it mean that the above theoretical arguments are incorrect and that such low mass Pop III stars have not been discovered only because of the difficulty in the observations?

Based on the results in the earlier section, we suggest that the first generation supernovae were the explosion of $\sim 20-130 M_{\odot}$ stars and some of them produced C-rich, Fe-poor ejecta. Then the low mass star with even $[\mathrm{Fe} / \mathrm{H}]<-5$ can form from the gases of mixture of such a supernova ejecta and the (almost) metal-free interstellar matter, because the gases can be efficiently cooled by enhanced $\mathrm{C}$ and $\mathrm{O}([\mathrm{C} / \mathrm{H}] \sim-1)$.

In contrast to the core-collapse supernovae of 20-130 $M_{\odot}$ stars, the observed abundance patterns cannot be explained by the explosions of more massive, $130-300 M_{\odot}$ stars. These stars undergo pair-instability supernovae (PISNe) and are disrupted completely (e.g., Umeda \& Nomoto 2002; Heger \& Woosley 2002), which cannot be consistent with the large $\mathrm{C} / \mathrm{Fe}$ observed in HMP stars and other C-rich EMP stars. The abundance ratios of iron-peak elements $([\mathrm{Zn} / \mathrm{Fe}]<-0.8$ and $[\mathrm{Co} / \mathrm{Fe}]<-0.2)$ in the PISN ejecta (Fig. 2; 
Umeda \& Nomoto 2002; Heger \& Woosley 2002) cannot explain the large Zn/Fe and Co/Fe in the typical EMP stars (McWilliam et al. 1995; Norris et al. 2001; Cayrel et al. 2003) and CS22949-037 either. Therefore the supernova progenitors that are responsible for the formation of EMP stars are most likely in the range of $M \sim 20-130 M_{\odot}$, but not more massive than $130 M_{\odot}$. This upper limit depends on the stability of massive stars.

\section{References}

Abel, T., Bryan, G.L., \& Norman, M.L. 2002, Science 295, 93

Audouze, J., \& Silk, J. 1995, ApJ 451, L49

Beers, T., \& Christlieb, N. 2005, ARAA in press

Bessell, M.S., Christlieb, N., \& Gustafsson, B. 2004, ApJ 612, L61

Cayrel, R., et al. 2004, A\&\&A 416, 1117

Christlieb, N., et al. 2002, Nature 419, 904

Christlieb, N., et al. 2004, ApJ 603, 708

Frebel, A., et al. 2005, Nature 434, 871

Galama, T., et al. 1998, Nature 395, 670

Hachisu, I., Matsuda, T., Nomoto, K., \& Shigeyama, T. 1990, ApJ 358, L57

Hamuy, M. 2003, ApJ 582, 905

Heger, A., \& Woosley, S.E. 2002, ApJ 567, 532

Hjorth, J., et al. 2003, Nature 423, 847

Honda, S., et al. 2004, ApJ 607, 474

Iwamoto, K., Mazzali, P.A., Nomoto, K., et al. 1998, Nature 395, 672

Iwamoto, K., Nakamura, T., Nomoto, K., et al. 2000, ApJ 534, 660

Iwamoto, N., Umeda, H., Tominaga, N., \& Nomoto, K. 2005, Science 534, 660

Mazzali, P.A., Iwamoto, K., \& Nomoto, K. 2000, ApJ 545, 407

Mazzali, P.A., Deng, J., Maeda, K., Nomoto, K., et al. 2002, ApJ 572, L61

McWilliam, A., Preston, G.W., Sneden, C., \& Searle, L. 1995, AJ 109, 2757

Nakamura, T., Umeda, H., Nomoto, K., Thielemann, F.-K., \& Burrows, A. 1999, ApJ 517, 193

Nakamura, T., Mazzali, P.A., Nomoto, K., \& Iwamoto, K. 2001a, ApJ 550, 991

Nakamura, T., Umeda, H., Iwamoto, K., Nomoto, K., et al. 2001b, ApJ 555, 880

Nomoto, K., Mazzali, P.A., Nakamura, T., et al. 2001, in Supernovae and Gamma Ray Bursts, eds. M. Livio et al. (Cambridge Univ. Press), 144 (astro-ph/0003077)

Nomoto, K., et al. 2003, in IAU Symp 212, A massive Star Odyssey, from Main Sequence to Supernova, eds. V.D. Hucht, et al. (San Francisco: ASP), 395 (astro-ph/0209064)

Nomoto, K., et al. 2004, in Stellar Collapse, ed. C.L. Fryer (Astrophysics and Space Science: Kluwer), 277 (astro-ph/0308136)

Norris, J.E., Ryan, S.G., \& Beers, T.C. 2001, ApJ 561, 1034

Ryan, S.G., Norris, J.E., \& Beers, T.C. 1996, ApJ 471, 254

Shigeyama, T., \& Tsujimoto, T. 1998, ApJ 507, L135

Sneden, C., Gratton, R.G., \& Crocker, D.A. 1991, A\&\&A 246, 354

Stanek, K.Z., et al. 2003, ApJ 591, L17

Tominaga, N., Umeda, H., \& Nomoto, K. 2005, in preparation

Turatto, M., Mazzali, P.A., Young, T., Nomoto, K., et al. 1998, ApJ 498, L129

Umeda, H., Nomoto, K., \& Nakamura, T. 2000, in "The First Stars", ed. A. Weiss etal. (Springer) (astro-ph/9912248)

Umeda, H., \& Nomoto, K. 2002, ApJ 565, 385

Umeda, H., \& Nomoto, K. 2003, Nature 422, 871

Umeda, H., \& Nomoto, K. 2005, ApJ 619, 427

Woosley, S. E., \& Weaver, T. A. 1995, ApJS 101, 181

Woosley, S.E., Eastman, R.G., \& Schmidt, B.P. 1999, ApJ 516, 788

Yoshii, Y. 1981, A\&A 97, 280

Zampieri, L., et al. 2003, MNRAS 338, 711 